\title{
Logic of Interval Uncertainty
}

\author{
Evgeny A. Kuzmin ${ }^{1}$ \\ ${ }^{1}$ Chair "Corporate Economics”, Ural State University of Economics, Ekaterinburg, Russia \\ Correspondence: Evgeny Kuzmin, Chair "Corporate Economics", Ural State University of Economics, Str. \\ March 8, 62, Ekaterinburg, 620144, Russia. Tel: 7-906-800-0070. E-mail: KuzminEA@gmail.com
}

Received: July 13, $2014 \quad$ Accepted: July 16, $2014 \quad$ Online Published: August 28, 2014

doi:10.5539/mas.v8n5p152 URL: http://dx.doi.org/10.5539/mas.v8n5p152

\begin{abstract}
The scientific category of uncertainty refers to that group of terms, an interpretation of which is not unambiguous and exact. In non-eliminability of the category soft content barrier there is an objective transition to the interval uncertainty. This research is an attempt to solve the issue of estimating the interval uncertainty based on methods of a logical analysis and a comparison. The approach presented by the paper is opposed to known methods of a mechanical selection of values following a given function. In the course of the research, there has been introduced a concept of the "tenversion uncertainty" for scientific use. Overall results obtained from the research allow calculating values of the interval uncertainty and assess their quality. The scientific competency of methods is achieved in theoretically tested solutions.
\end{abstract}

Keywords: interval uncertainty, fuzziness in economics, tenversion uncertainty, information entropy, uncertainty errors

\section{Introduction}

The intervality is an inherent property of parametric systems that function in a stochastic space. It is difficult to imagine that the economic or any other system possesses exact knowledge regarding a dynamics nature of its basic characteristics. Most often, this knowledge or, rather, ideas of distributed probabilities are approximate. In other words, they have a fuzzy nature. An indubitable fact is that "standard cases" for social systems are not typical. On the contrary, uncertainty conditions are typical. This conclusion does not require any proof and is present as a refrain in multiple academic papers, among which, for example, research by Parygin (1978), Leshkevich (1994), Afanasyev (1975) etc. are worth mentioning.

A quintessence of epistemological generalization is an idea saying that the inform ware for a managerial solution might look as a range from "the near empty to the almost comprehensive one" according to Afanasyev (1975). It seems that they are "almost" exact knowledge and understanding of a case at hand that do not allow the equally exact uncertainty to appear, the range of which is also large-scale starting from "almost unambiguous to an almost infinite number of alternatives" (Afanasyev, 1975). At the same time, regarding both well-known point entropy (an idealized standard), and its interval image, a starting point for scientific formalization and methodological classification of the uncertainty theory is a visualized assessment of their observability in the economic space. This research has been put in contrast to known approaches of mechanically selected uncertainty values on the interval. This research was designed to identify the uncertainty interval in a logical analysis of the function, take into account antinomy properties of entropy and consistency requirements.

\section{Literature Review}

Multiple academic papers have addressed complex systems in terms of the uncertainty. Scientists have paid their attention to solving various fundamental and applied tasks, where the entropy intervality is taken for granted, i.e. basic conditions, under which there is a progressing imaginative or real experiment. Thus, Kreinovich et al. (2011), Xiang (2007), Nguyen et al. (2008) have used terms of the interval uncertainty to refine estimates for statistical characteristics. Their Russian colleagues have made similar attempts. Panyukov and Latipova (2008) in interval estimates of the uncertainty will explain an equilibrium in the J. von Neumann's model of an expanding economics. Thus, numerous aspects of social and economic interaction strongly emphasize an interval nature of these phenomena development, which is quite natural.

A separate corpus of scientific papers deals with issues of network optimization for complex systems. The interval uncertainty therein is inter alia a consequence of incomplete measurements, such as in the paper by Shen 
et al. (2011), where with the interval-matrix approach there is one of the earliest attempts to describe the uncertainty of an internal state of the network; or as a result of the uncertainty (or incompleteness) that relates to a constraint force between nodes of the system. Deepening a range of problems, Shang (2011) comes closer to an answer to the similar question, but for scale-free networks yet. A main emphasis is made on exploring sustainability influenced by the "gray information", i.e. the information comprehensive in its scope of objects (nodes within the system), but inexact. It is this inexactness that produces the interval of fiducial perception. A certain interest under this direction is generated by research in interval-dependent and interval-independent complex dynamical networks. There will be name worthy papers by Li and Yue (2010), as well as Li, Wong and Tang (2012), etc. Both publications do not only point out to an influence of the subordination factor between nodes of the network, directly preconditioning dependences, but also the time factor. Transitions from one mode to another with a certain probability produce mixed time delays aggravating the system uncertainty.

The paper by Fortin, Zieliński, Dubois and Fargier (2010) has recently summarized the issue of the interval uncertainty for the theory of complex networks. This research revises the issue of PERT-scheduling with the incomplete information, seemingly well-known following the Kelley's critical path method (1961). Oddly enough, but vagueness in time distribution to execute tasks, a moment to start the ones and finish the others have for a long period of time remained outside the research attention in terms of the interval uncertainty. There is a particular emphasize on the fact that actually distribution of probabilities allows simulating variability of repetitive tasks, but not the uncertainty due to a lack of the information (e.g., Dubois, Prade and Smets (1996), Ferson and Ginzburg (1996), et al.). Only as an approximate solution to the mentioned range of problems we consider approaches by Chanas and Kamburowski (1981), Hapke, Jaszkiewicz and Słowiński (1994), Loostma (1997), McCahon and Lee (1988), as well as Prade (1979) and Rommelfanger (1994).

However, the uncertainty cannot and must not only act as a condition, under which there are economic or other processes in progress. With insufficiently elaborated issues of calculating the interval uncertainty in methods of a logical choice, their methodological support is a fundamental scientific challenge. A solution to this challenge is in line with a proposed viable alternative, where initial terms of the uncertainty have not been set by parameters of a case, but become an independent object to be scientifically explored.

The first step to compile in an estimate way the interval uncertainty is a probability. It is an available range of probabilities for each alternative to be implemented in their assembly that leads to the entropy of a dimension value to appear. On the other hand, with apparent simplicity of the approach, implying an everyday substitution of probability values, boundaries of the interval uncertainty lie in a different plane. A cause for this lies in a property of antinomy.

An antinomy property of the uncertainty, whereby the entropy of a single instance may be located in a negative area (for more details see Kuzmin (2012)), is based on a displacement and asymmetry of the uncertainty function. The antinomy nature explains all the complexity of logical calculus for the interval uncertainty, when given boundaries of the certainty in the totality of values contain the internal range of the interval uncertainty. It is a search for it that this research is focused on. As a result, the above-mentioned antinomy specifics makes three common cases that call for matching: a full match and overlap, divided into the matching right and left parts. As a result, two combined area appear: the first one is at a distance from the lowesr limit up to 1/e (basis of the natural logarithm); the second, on the contrary, is at a distance from 1/e up to the top limit of the probability (see Figure 1). An upper extremum of the entropy (calculated per alternative) corresponds to the permanent constant value of the probability, causing both non-linearity and function displacement at the same time. Thus, a major scientific challenge shapes itself, when the available interval of the probability that characterizes case fuzziness, within its boundaries does not correspond to the uncertainty interval.

Another, by no means unimportant, issue is the content of probability intervals. Surely, the range might be both continuous and discontinuous, and dotty. Recognising the interval as a continuous one is an assumption for this paper. In this regard, the view of Kyburg (1989) and Neapolitan (1996) is amazing. They share an opinion that probability intervals can always be reduced to point wise values. However, to our mind, this is not always the case. Depending on requirements of refinement and measurement accuracy as such, point wise values cannot be applied. There are natural barriers preventing decomposition of a probability line into point wise factors. Papers by Kyburg (1989) and Neapolitan (1996) do not mention this, but each probability only appears and lives within the framework of an adopted coordinate system, where a numerical order of the probability has a crucial value. As a result, one might reasonably state that the point look cannot be a worthy replacement for the linear probability (unbroken), even if it had an interval limit. A disclaimer expressed by some scientists, such as Sternin and Shepelev (2012), saying that "...an interval estimate contains a continuum of possible point wise implementations" only partially resolves existing contradictions in views. 
Halpem and Fagin (1992) at the same time pay attention to the theory of probabilities, which explains a mechanism to join the interval. Later even Yager and Kreinovich (1999) suggested and gave grounds for a formula of statistical estimates for the so-called secondary interval. It is indicative that to get such estimates one needs to know the distributive law, to identify which within a fuzzy set "with a multi-valued scale of truth" (Borisov et al., 1989) is at least difficult. From Parsons's (2001) point of view, it proves again an ambiguous nature of the uncertainty, for which no specific characteristic and quite accurate values are typical. Somewhat earlier, Ang and Tang (1975) came to such conclusion, when they reasonably assumed that "the information can be often expressed in terms of the lower and upper variables of the limit". This implies that the probability like the information possesses constraints, going beyond which can be a trouble. One might say that thereby the uncertainty should remain unknown to some extent.

Returning to Parsons's ideas (2001), it is worth paying attention to his statement of the interval interpretation, "the presentation interval, whether this is a probability or not, is intuitively compelling in making the inexactness clear." An undisputed nature of the statement should be read with a limitation that clarity appears in understanding the essence of arising divergences. At the same time, Parsons's considers the interval uncertainty a result of the fuzzy probability manifested itself. Therefore, major efforts by researchers address the issue of "how to make assumptions of the partial information that have not been clearly defined." Thus, making corrections to the interval uncertainty or in other words reducing it to the point uncertainty to some extent, some scholars see in processing the information itself. And according to Parsons's statements, "these systems can cope with a situation... even in the absence of necessary and partially defined probabilities..." It seems that such the way would not be without results, though manipulations with the interval uncertainty to optimize management decisions taken could be more efficient. After all, generating new information to narrow down a gap of the uncertainty is linked, on the one hand, to arising ambiguity of new data, and on the other - to the complexity of artificial refinement of the state. A compromise when dealing with the source information is achieved in a revision of probability intervals. This conclusion made by Loui (1986) is shared by Parsons's and consistent with the authors' hypotheses regarding the interval normalization.

Considerations on a heuristic evaluation of probability distribution are separately worth looking at. Problems concerned in the work by Beliaev and Makarov (1974), are solved using a principle of the maximum entropy as a common method for these purposes, which, nevertheless, with all internal consistency of the approach, does not avoid a number of disadvantages. Dichotomy in a search for favourable and hence inter alia labelling unfavourable distribution is counterbalanced using the confidence interval, when a case and a condition are specific enough, but retain their development uncertainty. At the first glance, such a conclusion sounds like a kind of paradox. However, specificity lays in a structure and probabilities of alternatives, which have predefined values. The confidence interval mostly influenced by the idiosyncratic "errors of uncertainty" (in terms of the alpha and beta errors) seems to be a kind of prologue to studies in distribution fuzziness. The introduction to the research methodology refines meaningful aspects differentiated by types of the uncertainty errors. To the author's mind, one needs to distinguish between alpha-, beta-and gamma-errors of the uncertainty (so-called standard errors), that set out possible errors in perception and, mainly, in its quantity assessment. We should clarify some of their characteristics. An essence of the alpha- error depends on making a set of alternatives recognised as basic. Subjective deviation from multiple possible outcomes from certain scenarios (hypotheses) shows the additional uncertainty, a value of which is calculated by relating two states - actual and conditionally ideal. The Beta-error of the uncertainty goes to a quality side of alternatives and implies fuzziness in defining likelihood or frequency for each outcome. A nature and an extent of different errors' participation in assessing a numerical value of the uncertainty are presented with more details in other research, e.g. Avdiysky and Kuzmin (2013).

\section{Methods}

\subsection{Reducing Probabilities}

Stochastic dynamics within complex systems in fuzzy distribution sets certain limits, i.e. requirements to intervals of the alternatives' probability. The single instance, being internally consistent by formal unity criteria must be harmoniously integrated into an overall ensemble. This suggests a need in a cross-validation of alternatives' probabilities, designated within the research as the convergence requirement or convergence condition. Objectively available limitations related to the information and the uncertainty also lead to this. Starting from a paradigm objective, offered by Lindley (1987) (not without assumptions according to Elishakoff and Ohsaki (2010)), an idea of drafting a "single instance" with acceptable alternatives, that is such a hypothetical event, which has its unitary probability in its possible implementation to assess and calculate the uncertainty. A range of intervals in this case is adjusted by excluding those probabilities that within the ensemble 
of alternatives go beyond pragmatic affordability.

The above-mentioned conventionally ideal case, regarding to the alpha-error calculation, is also suitable to explain the proposed condition, “... based on the idealized interval adjustment, there is a provided tool to expand coverage to include its true [frontiers - scholium]" (Spall, 2002). Its essence lies in a principle check of extreme instance probabilities - those of the upper and lower thresholds. Scientific grounds for a methodological imperative of probabilities convergence (or reducing) is based on the information coverage. Completeness of alternatives should be a hypothetical case, which occurrence probability is equal to one. Verification of ranges assumes both their targeted expansion, and contraction. Herewith, a process of such a transformation can go either from the upper or lower limit of the probability interval.

Hereby, there are actually only a few given initial parameters set: a number of alternatives making up the ensemble and the probability matching them, though either the maximum or the minimum one. Only to confirm this rule, there are exceptions, when initial probability intervals meet a "reducing" requirement. By the way, Elishakoff and Ohsaki (2010) ground a similar condition in the form of optimization and anti-optimization models, that stand for options of best and the worst scenario approaches. Drawing parallels between conclusions and ideas by Elishakoff and Ohsaki (2010), one can quite reliably refer to the optimization model as an approach of a noncompliant attitude towards the uncertainty. An opposite picture concerns the anti-optimization model, where there is a tolerant perception of the entropy. In both cases, models operate with probabilistic values, which, even with expanding or contracting intervals require reducing. The logic of probability intervals adjustments is presented below.

Taking $a_{1}$ and $a_{2}$ for the lower and upper bounds of the $p_{i}$ probability range, respectively for the i-th alternative within the hypotheses ensemble, one can represent a mathematical probability convergence (or reducing) record, that looks like as follows:

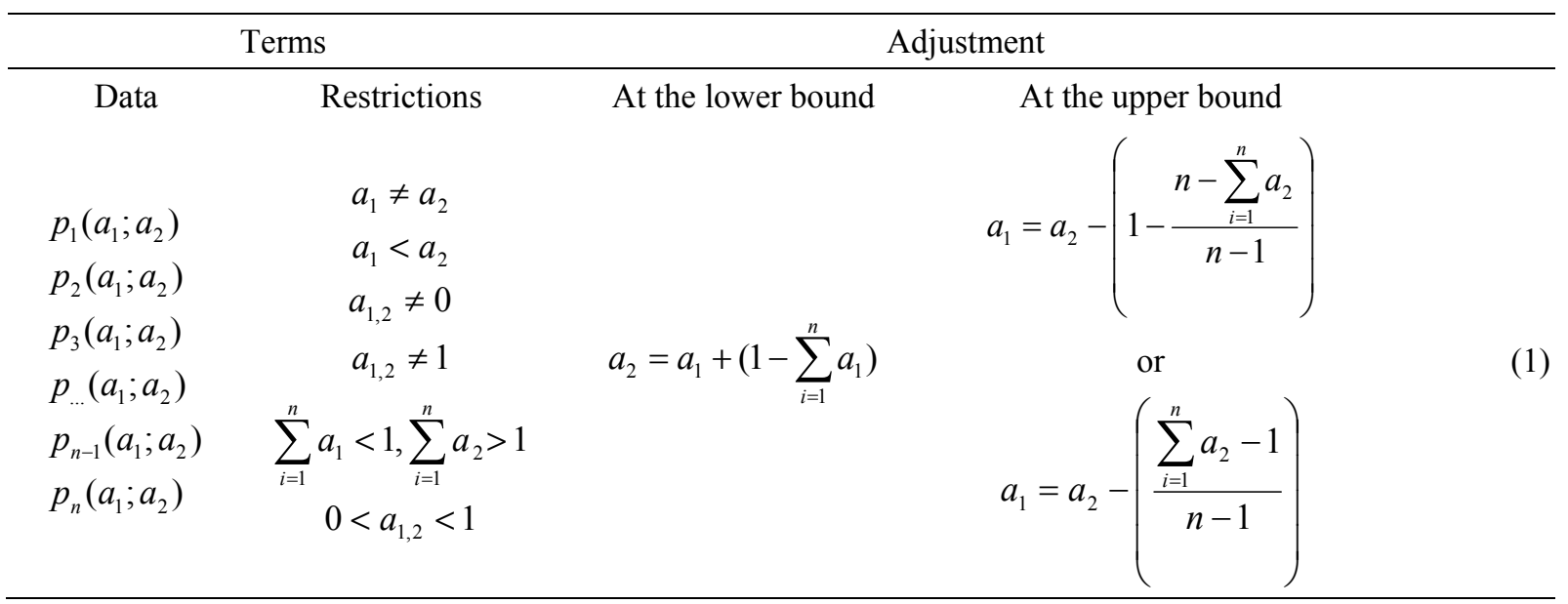

It is amazing that for all cases of the probability interval reducing there is a coherence equation identified by the research. Its statement says, the sum of probabilities for the lower bound is equal to a converted value of the sum of probabilities of the upper bound, which depends on a number of alternatives -

$$
\sum_{i=1}^{n} a_{1}=\frac{\sum_{i=1}^{n} a_{2}-1}{n-1}
$$

The abovementioned assumes that initially set probability ranges might be mutually non-coherent. Reducing probabilities solves this problem. A contraction process results in making the coherent probabilistic logic. But methodological difficulties do not end at this point. Here one should remind that gaps in hypotheses distribution do not allow getting reliable estimates, i.e. the uncertainty would be a priori incomplete without taking into account the additional complexity of the choice. Guo and Tanaka (2007), with their ideas concerning mostly the economic profit, make a fair conclusion that "an optimal order is obtained based on a partially ordered array of interval expectations" (Guo and Tanaka, 2010). 


\subsection{Search for "Single Instance" Criteria. Combination Uncertainty}

Having obtained representative intervals for probabilities per each alternative included in the uncertainty area, we have a scientific challenge of a different kind, a solution to which involves a search for equivalent or close options on a contour of the entropy function.

Apodictic ideas of optimizing the uncertainty situation suggests that with quantity similarity or equality of values in intervals of different alternatives there is a possibility of cases combination, where a level of the interval uncertainty remains unchanged. As a result, one can reasonably say of heterogeneity within the interval entropy. On the one hand, this results from the function specifics. On the other hand, this results from an objective need to "gain to fit" the probability, as relevance of the interval entropy estimates is provided by obtaining the single instance, which in its turn cannot be only composed of the lower or the upper boundaries of the range of alternative probabilities.

A single-directed search for a range of the interval uncertainty (by reducing the probability) makes us only a bit closer to a founded hypothetical scenario. A conceptual approach to an assessment of the interval uncertainty, proposed by the paper, assumes a two-level comparison: the first level influences private alternatives, while the second one aims at probabilities harmonization, when the single event is made with upper and lower bounds of the uncertainty. An outcome of assessment calculations is the dual uncertainty that in the first case is referred to as the uncertainty of combinations (intermediate value), while in the second case as the actual interval uncertainty.

One can trace overlapping "surplus" or "shortage" in possibilities up to a single constant calculating the uncertainty of combinations. A starting point for this is a collatable analysis of alternatives. The technique allows looking at those alternatives, which within the reduced probability of intervals (in threshold values) have already had some maximal or minimal uncertainty, but have not still met a requirement of the single instance. Hereinafter, a search for the interval uncertainty will be focused on a particular combination of probabilities. At the same time, a structure of hypotheses, according to the technique, is differentiated into four groups depending on a correspondence between the upper and lower threshold of probabilities per alternative and the given conditions (3). It is worth saying again that the total moment probability for the uncertainty of combinations might be either over or lower than one (it can seldom have values equal to one).

$$
\begin{array}{r}
H_{\text {extracomb }}^{\max }=H(A)+H(B)+H(C)+H(D) \\
H_{\text {extra comb }}^{\max }=\left[\begin{array}{l}
a_{1}<\frac{1}{e}, a_{2} \geq \frac{1}{e}: H(A)=m\left(-\frac{1}{e}\right) \log _{b}\left(\frac{1}{e}\right) \\
a_{1}<\frac{1}{e}, a_{2}<\frac{1}{e}: H(B)=\sum_{k=1}^{k}-a_{2} \log _{b}\left(a_{2}\right) \\
a_{1}>\frac{1}{e}, a_{2}>\frac{1}{e}: H(C)=\sum_{l=1}^{l}-a_{1} \log _{b}\left(a_{1}\right) \\
a_{1}=\frac{1}{e}, a_{2}>\frac{1}{e}: H(D)=t\left(-\frac{1}{e}\right) \log _{b}\left(\frac{1}{e}\right)
\end{array}\right]
\end{array}
$$

where $H_{\mathrm{extracomb}}^{\max }$ is the maximum uncertainty of combinations; $H(A), H(B), H(C), H(D)$ stands for component grouped parts of the uncertainty; $a_{1}, a_{2}$ stands for the lower and upper bounds of the range of probabilities of the i-th alternative; $m, k, l, t$ is a number of matches under the terms of calculation.

A situation is somewhat different with finding the minimum uncertainty of combinations. With the arched function, the minimum of the i-th alternative entropy can be located both on the right part (increasing area), and on the left (decreasing area) part (see Figure 1). Herewith, it is worth paying attention to displacement of the function itself. It is necessary to make a few remarks concerning this. 


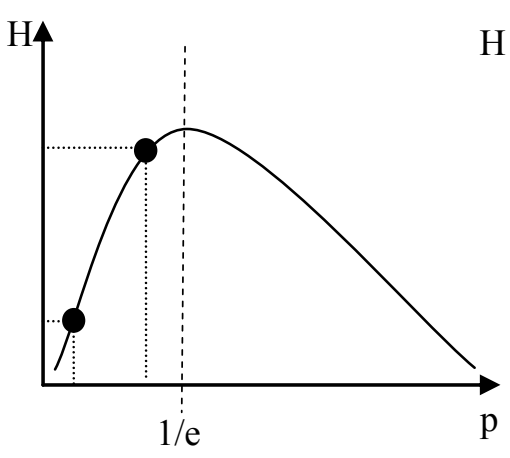

a)

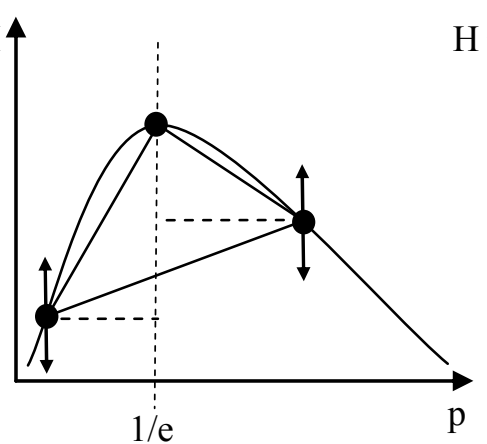

b)

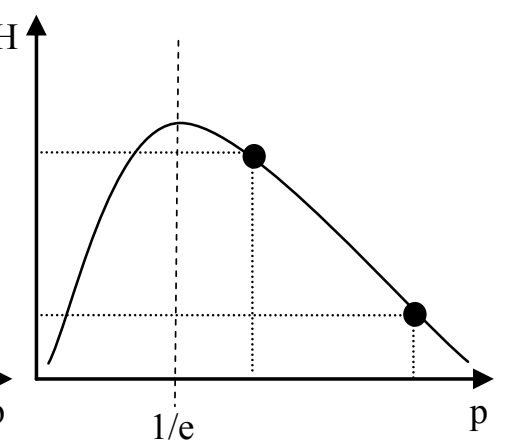

c)

Figure 1. Common cases and fields of matched probabilities when assessing the interval uncertainty

in decomposition of private alternatives: a) match on the right side of probabilities distribution; the minimum and maximum uncertainties fully correspond with boundaries of the probability interval; b) complete match; identifying the probability is associated with an issue of uncertainty comparison, when the maximum value is known attributable to $1 / \mathrm{e}$, whereas the minimum is unknown, which locates either to the right, or to the left of the central axis; c) match in the left part of the probability distribution; the maximum and minimum uncertainties are inversely proportional to relations between probability boundaries.

A main thesis is that different values of the probability can make the identical uncertainty. The approach, proposed by the author, proves that firstly with the identical uncertainty deviations of probabilities from a central axis are non-equal. Secondly, these probabilities make the dual minimum of the uncertainty, but in calculations, only one of them is taken into account, i.e. that one that to the more extent makes a set of alternatives closer to the single instance. Thirdly, deviation on the right side $(\beta)$ in a function polygon is always over than deviations on its left part $(\alpha)$. Hence, formalized terms for the observable minimum of the uncertainty are complemented with a special factor $\left(k_{\alpha, \beta}\right)$, which accentuates a move from a case of the entropy identity (equality) in case of a numerical non-equality of probabilities for considered alternatives.

Based on conclusions presented, a process to obtain the minimum uncertainty of combinations looks like the following:

$$
\begin{gathered}
H_{\text {extra comb }}^{\text {min }}=H(A)+H(B)+H(C)+H(D)+H(C \vee D) \\
H_{\text {extra comb }}^{\text {min }}=\left[\begin{array}{l}
a_{1} \geq \frac{1}{e}, a_{2}>\frac{1}{e}, a_{1}<a_{2}: H(A)=\sum_{m^{\prime}=1}^{m^{\prime}}-a_{2} \log _{b}\left(a_{2}\right) \\
a_{1}<\frac{1}{e}, a_{2} \leq \frac{1}{e}, a_{1}<a_{2}: H(B)=\sum_{k^{\prime}=1}^{k^{\prime}}-a_{1} \log _{b}\left(a_{1}\right) \\
a_{1}<\frac{1}{e}, a_{2} \geq \frac{1}{e},\left(a_{2}-\frac{1}{e}\right)>\left(\frac{1}{e}-a_{1} k_{\alpha, \beta}\right): H(C)=\sum_{l^{\prime}=1}^{l^{\prime}}-a_{2} \log _{b}\left(a_{2}\right) \\
a_{1}<\frac{1}{e}, a_{2} \geq \frac{1}{e},\left(a_{2}-\frac{1}{e}\right)<\left(\frac{1}{e}-a_{1} k_{\alpha, \beta}\right): H(D)=\sum_{t^{\prime}=1}^{t^{\prime}}-a_{1} \log _{b}\left(a_{1}\right) \\
a_{1}<\frac{1}{e}, a_{2}>\frac{1}{e},\left(a_{2}-\frac{1}{e}\right)=\left(\frac{1}{e}-a_{1} k_{\alpha, \beta}\right): H(C \vee D)=\sum_{w^{\prime}=1}^{w^{\prime}}\left(-a_{1} \vee-a_{2} \log _{b}\left(a_{1} \vee a_{2}\right)\right.
\end{array}\right]
\end{gathered}
$$

where $H_{\text {extra comb }}^{\min }$ is the minimum uncertainty of combinations; $m^{\prime}, k^{\prime}, l^{\prime}, t^{\prime}$ is a number of matches under the terms of calculation.

\subsection{Interval Uncertainty}

A scientific search for the interval uncertainty goes along with a verification of previously obtained moment probabilities for the hypothetical event, which appear in terms of calculation concerning the maximum (3) and 
the minimum (4) uncertainty of combinations. Probabilities make a matrix, which is a basis for a secondary comparison. In particular, for the maximum, an equiprobable instance becomes such a comparison criterion $(\mathrm{p}=1 / \mathrm{n})$.

\subsubsection{Maximum of the Interval Uncertainty}

The maximum interval uncertainty, as in other calculation options, assumes making an acceptable scenario. To the author's mind, the process is largely connected to unification of manifested alternatives (equal probability). Herewith, as it is known, a numerical limit for the uncertainty is reached. All arithmetic of the method is divided into a number of stages, while a result of their implementation is an approximate estimate. A disclaimer on an approximate nature of calculations is due to the nonlinear dynamics of the uncertainty, allowing errors that is actually inherent to the standard error area.

1) The first step to obtain the actual uncertainty includes gradually converging probabilities. At this stage, the maximum entropy antinomy $(p=1 / \mathrm{e}$ ) opposes to the equal probability maximum. By comparing the previously obtained matrix for the uncertainty of combinations and the decrease delta, it is possible adjust to some extent the additive sum and take the situation closer to the single equiprobable instance. Indicative parameters, i.e. the decrease delta and the growth delta, are measured with the module. Actually, each of them shows possibilities of a change to the probability (per alternative), depending on set intervals that correspond to the principle of convergence, towards the single event. An essential assumption is an idea of elimination, saying that to reduce to the single event one needs to change the given probability (within intervals) for only one alternative (such calculation is made for all the alternatives).

$$
P_{\text {real (interim) })_{i}}^{\max }=\left[\begin{array}{l}
\left(p_{i}^{\operatorname{maxcomb}}-\Delta p_{i} \downarrow\right) \leq \frac{1}{n}: \frac{1}{n} \\
\left(p_{i}^{\operatorname{maxcomb}}-\Delta p_{i} \downarrow\right)>\frac{1}{n}: p_{i}^{\operatorname{maxcomb}}-\Delta p_{i} \downarrow
\end{array}\right]
$$

where $P_{\text {real(interim) }}^{\max }$ is an intermediate probability matrix for the maximum actual uncertainty in interval measurement; $p_{i}^{\max c o m b}$ is the probability of the alternative, corresponding to the maximum of the uncertainty of combinations; $\Delta p_{i} \downarrow$ is the probability decrease delta.

2) Mostly, the additive sum of the smoothed-out probability according to (5) to the full extent have not correlated proposed requirements. However, at the same time, new logic regularities appear that determine subsequent conversions. An obvious consequence is such a situation, in which there is a need for adjustment. With the alternative probability exceeding $1 / \mathrm{n}$ value (such difference is accumulated), a reduction value appears that makes an excess probabilistic load. Its smoothing-out happens at the next stage, where, firstly, those alternatives should be clarified, where probabilities of their occurrence are over than $1 / \mathrm{n}$. Secondly, one needs to use the upper and lower bounds of the set range to evaluate a potential of possible variations in values. For this, we introduce a number of variable and conditional parameters, intended to simplify perception of the calculations logic. All comparative conversions and calculations occur at the second methodological step.

A sequence of calculations can be presented as the following formalization:

$$
y_{1}^{\max }=\left[\begin{array}{l}
P_{\text {real (interim 1) }}^{\max }-\frac{1}{n}=0: a_{1} \\
P_{\text {real (interim 1) }}^{\max }-\frac{1}{n} \neq 0: P_{\text {real (interim 1) }}^{\max }
\end{array}\right], y_{2}^{\max }=\left[\begin{array}{c}
P_{\text {real (interim 1) }}^{\max }-\frac{1}{n}=0: a_{2} \\
P_{\text {real (interim 1) }}^{\max }-\frac{1}{n} \neq 0: P_{\text {real (interim 1) }}^{\max }
\end{array}\right] .
$$

Then with similar conditions of variables $y_{1}^{\max }$ and $y_{2}^{\max }$ one needs to identify similar values and differentiate in pairs differences between them (matrix $r_{1}^{\max }$ and matrix $r_{2}^{\max }$ ).

$$
r_{1}^{\max }=\left[\begin{array}{l}
\mathrm{y}_{1}^{\max }=y_{2}^{\max }: y_{2}^{\max } \\
\mathrm{y}_{1}^{\max } \neq y_{2}^{\max }: 0
\end{array}\right], \quad r_{2}^{\max }=\left[\begin{array}{l}
\mathrm{y}_{1}^{\max }=y_{2}^{\max }: 0 \\
\mathrm{y}_{1}^{\max } \neq y_{2}^{\max }: y_{2}^{\max }
\end{array}\right]
$$

The next variable $\left(U^{\max }\right)$ is an indicator that tracks those alternatives, the probability of which will change in contrast to a number of hypotheses made earlier on the intermediate matrix (5). The author's approach involves a method to average remaining hypotheses, as only in this case the uncertainty is maximum. Herewith, so-called 
unchanging alternatives become such not so much because of assessment preferences of the uncertainty of combination, as of being a result of exact compliance with interval analysis requirements.

$$
U^{\max }=\left[\begin{array}{l}
r_{2}^{\max }=0: r_{2}^{\max } \\
r_{2}^{\max } \neq 0: r_{2}^{\max }-\frac{\sum_{i=1}^{n} r_{1}^{\max }+\sum_{i=1}^{n} r_{2}^{\max }-1}{\sum r_{2}^{\max }\left(r_{2}^{\max }>0\right)}
\end{array}\right] .
$$

3) The resulting matrix (8) completes the second step of intermediate comparative estimates and leads to the final stage, where there will be obtained a quantitative evaluation of the approximate maximum uncertainty for the interval. More precisely, it leads to determination of the moment probability, with which such the uncertainty may occur.

Thus, the matrix of the actual probability (the upper bound of the interval) makes such an instance, which was before referred to as a single, that is, in fact, the main requirement to comprehensive estimates for the uncertainty.

$$
\begin{aligned}
& P_{\text {real }}^{\max }=\left[\begin{array}{l}
\sum_{i=1}^{n}\left(P_{\text {real }(\text { (interim })_{\mathrm{i}}}^{\max }-\frac{1}{n}\right)=0: \frac{1}{n} \\
\sum_{i=1}^{n}\left(P_{\text {real }(\text { (interim })_{\mathrm{i}}}^{\max }-\frac{1}{n}\right) \neq 0: r_{1}^{\max }+U^{\max }
\end{array}\right] \\
& \text { wherein } \sum_{i=1}^{n} P_{\text {real }_{i}}^{\max }=1 \text {. }
\end{aligned}
$$

At the same time, the explained approach with all advantages and its logical structure operates with a prerequisite that cannot avoid criticism. We mean averaging alternatives: even if variable probabilities of alternatives are only in one of the function areas, it is not possible to argue that their values placed at equal distances have uncertainty levels placed at the same distances between them.

However, strong arguments have remained in favour of the averaging idea (Note 1). The error in calculations caused by the function linearization is not as weighty to reject such an assumption. Previously marked features of the entropy function show that significance of the numerical error in probability averaging increases as we approach the extremum, or more precisely, a limit of the uncertainty. It is known that prevailing one or more alternatives, in which a frequency of manifestations is not identical, leads to occurrence of the case certainty. Averaging, on the contrary, makes the uncertainty and makes the choice more complex. It is quite possible that to maximize the uncertainty, some alternatives not yet fixed within the scenario should have the probability equal to $1 / n$, whereas other alternatives will act as a compensating factor with the different probability for a holistic combination of options to make the single unstance. Based on these and other reasons put forward in the course of the research, the methodical approach has kept an assumption of probability averaging understanding that an estimate of the maximum interval uncertainty is only approximate and different by a value of a corresponding error.

\subsubsection{Minimum of the Interval Uncertainty}

Making a pause at a point of giving grounds for a calculation of the maximum uncertainty intervally interpretated, one should address calculations for the range lower bound. Approaches to their estimate differ due to antinomy properties of the function. It is worth saying again that the minimum of the uncertainty is ambiguous and under specific accident circumstances, it is dual. Thus, the approach to the assessment assumes well-founded research in several basic conditions that allow eliminating duality contradictions.

It seems that the easiest condition for a differentiated comparison is convergence of probabilities. Based on a need to construct the single instance, criteria for the convergence condition are grouped within three options at least. The first implies that the additive sum of probabilities of alternatives included in the minimum uncertainty of combinations is equal to one. Herewith, further conversions and calculations are no longer required; the minimum actual uncertainty has been already achieved. The second and the third options are based on the opposite saying that the sum of hypotheses probabilities is inconsistent with the single instance, and hence the probability is either over, or lower than one. One needs to note that only one option reflects an actual situation with the uncertainty. To observe logic in the method presentation, it is useful to present mathematical formalization and explanation for each of them. 
1) According to the abovementioned, refinement of the first option is only required because of a holistic nature of the method. Here, the sum of probabilities with the minimum uncertainty of combinations (4) have been equal to one that allows founded assuming on obtaining the interval entropy minimum herewith. A verification of the instance is rather formal.

$$
\sum_{i=1}^{n} p_{i}\left(H_{\text {extra comb }}^{\text {min }}\right)=1 \mid \Rightarrow p_{i}\left(H_{\text {extra comb }}^{\min }\right)=p_{i}\left(H_{\text {real }}^{\text {min }}\right)
$$

A different picture emerges in a case when the additive sum of probabilities according to (4) is not equal to one. There is an objective need in value convergence and hence in covering its surplus as in the third option or a shortage as in the second option. It is worth referring to each option.

2) A search for the minimum of the interval entropy in the second option goes towards matching the uncertainty of critical levels (we mean multiple values of probabilities, i.e. of the lowest and upper boundaries of the interval, as well as of the variable $1 / \mathrm{n}$ and the constant $1 / \mathrm{e}$ ) and the uncertainty of combinations for different hypotheses. A result of this is a notation of those alternatives, where the probability becomes as a kind of a fixed one. Its value gets one of values of a critical level, mostly of the lowest bound of the probabilities range. Remaining hypotheses (alternative) highlight a need in frequency "finish gathering" up to the single instance. A solution to this task lies in an area of identifying the so-called first-match (Note 2).

Formalizing the approach for the second option, as in other examples given above, involves calculating intermediate variables to fix various logical search requirements. According to the abovementioned, such an indicator includes the first match criteria. However, it follows reconfiguration of probability critical values. A mathematical expression for this procedure applied for this type of calculations is as follows:

$$
p_{\text {lower limit }}^{\min }=\left[\sum_{\mathrm{i}=1}^{\mathrm{n}} p_{\text {extra comb }_{\mathrm{i}}}^{\min }<1: p_{\text {extra comb }_{\mathrm{i}}}^{\min }\right], \quad p_{\text {upper limit }^{\min }}=\left[\sum_{\mathrm{i}=1}^{\mathrm{n}} p_{\text {extra comb }_{\mathrm{i}}}^{\min }<1: a_{2}\right] .
$$

New thresholds for ranges combine in themselves a value for the resulting probability for the minimum uncertainty of combinations and the upper probability of the specified interval. A need in such a transformation is caused by an objective need to cover a frequency deficit. Returning to the first match criterion, it will be useful to clarify the indicator figure $\left(q^{\mathrm{min}}\right)$, based on which the criterion is defined:

$$
q^{\mathrm{min}}=\left[\begin{array}{l}
\left(p_{\text {lower limit }}^{\min } \log _{b} p_{\text {lower limit }}^{\min }-p_{\text {upper limit }}^{\min } \log _{b} p_{\text {upper limit }}^{\text {min }}\right)>0: p_{\text {lower limit }}^{\min } \log _{b} p_{\text {lower limit }}^{\text {min }}-p_{\text {upper limit }}^{\min } \log _{b} p_{\text {upper limit }}^{\min } \\
\left(p_{\text {lower limit }}^{\text {min }} \log _{b} p_{\text {lower limit }}^{\text {min }}-p_{\text {upper limit }}^{\text {min }} \log _{b} p_{\text {upper limit }}^{\text {min }}\right) \leq 0: \text { error }
\end{array}\right] .
$$

The presented matrix (12) is an estimate of a difference between uncertainties according to (11). A search for the first match is performed with the minimum value among obtained values. It is this alternative, for which there will be acceptable an adjustment to the probability. Herewith, other hypotheses have remained in the same, unchanged form. Thus, a final structure of the alternatives probability to calculate the minimum of the interval certainty is calculated observing the following way:

$$
\begin{gathered}
p\left(H_{\text {real }}^{\text {min }}\right)=\left[\begin{array}{l}
q_{i}^{\text {min }}=\min ^{1}\left\{q^{\text {min }}\right\} \mid<q_{i}^{\text {min }}>_{1}: p_{\text {lower limit }}^{\text {min }}+\left(p_{\text {upper limit }}^{\text {min }}-a_{1}\right) \\
q_{i}^{\text {min }} \neq \min ^{1}\left\{q^{\text {min }}\right\}: p_{\text {lower limit }}^{\text {min }}
\end{array}\right], \\
\text { wherein } \sum_{i=1}^{n} p_{i}\left(H_{\text {real }}^{\text {min }}\right)=1,
\end{gathered}
$$

where $\min ^{1}\left\{q^{\min }\right\}$ is the first order minimum within $q^{\min }$ array of values.

3) The third option to estimate the minimum uncertainty comes from the fact that the additive sum of probabilities for hypothetical accident alternatives, on the contrary, is over one. This has resulted in an uncompromising search for the minimum entropy. However, the underlined difference does not underplay importance of using the method of the "first match". In both cases, they identify such an alternative, which is less in its uncertainty inflection. In other words, such the hypothesis is remarkably consistent in its uncertainty dynamics. To say more, in its low minimum entropy spread within the given range. Because the uncertainty of 
combinations has been an extreme manifestation of the minimum or maximum uncertainty, but has not yet been the single instance, this method seems to be justified and scientifically grounded.

To smooth categoricity in choosing the momentum probability value, possible because of equal ranges between alternatives, the third option assumes using not only the method of the "first match", but also the "the last match" (Note 3). Moreover, the method of the "first match" becomes a more rigid form as of the only match. Each method demarks possibly repeated or identical ranges of hypotheses probabilities. Assuming that alternatives are mutually distinct, the first match method, in fact, shows a single hypothesis, for which there will be appropriate a change to the probability with minimal consequences. A reverse situation occurs when some or all of alternatives are similar. A need occurs to reduce probabilistic tension by influencing only some of these alternatives - apart from the first until the last match. However, with absent identically similar hypotheses there is an exception to rules. Obviously, the set of alternatives is not homogenous; it can include hypotheses with the minimum uncertainty in both the right and the left part of the function. In this, a need occurs to apply these two methods in combination. Their incorporation marks a new synthetic method, i.e. the method of "inter congruence". Adjustments within it only goes for those alternatives that follow according to the minimum criterion specified with index $q^{\min }$ (Note 4). This occurs until the moment probability sum for the scenario is equal to one. A limit number of such points depends on a number of numeric values $q^{\min }\left(W_{q^{\min }}\right)$, which are reduced by an alternative. Decrease in a number of selected hypotheses depends on maintaining the probability balance. Its essence is in leaving a "free alternative", which takes probabilistic values based on compared uncertainties. Such alternative is recognized as the last of the ranked $\left(\operatorname{Rank}_{i}\right)$ set of values for the $q^{\min }$ indicator (ascending from 1 to $\mathrm{n}$ ). It is important that ranking goes for all the hypotheses without no repetitions in the rank value.

Based on the assumptions made above, the initial probability of boundary values in the third option also faces changes. Since the additive sum of probabilities is within a zone of excess tension, the lowers limit replaces the top one: we consider a possibility for optimal reducing in probabilities. Then, we obtain limits as follows:

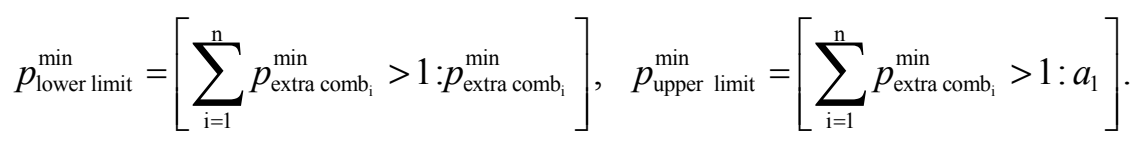

Herewith, one should distinguish probabilities (14) and boundary values obtained as expressed in the equation in the second option (11). Their identical notation has been kept due to an exclusive situation, when a calculation is only possible under one of options.

The following equation establishes the probability-decrease delta (s) per alternative regarding the previously established hypotheses within the minimum uncertainty of combinations.

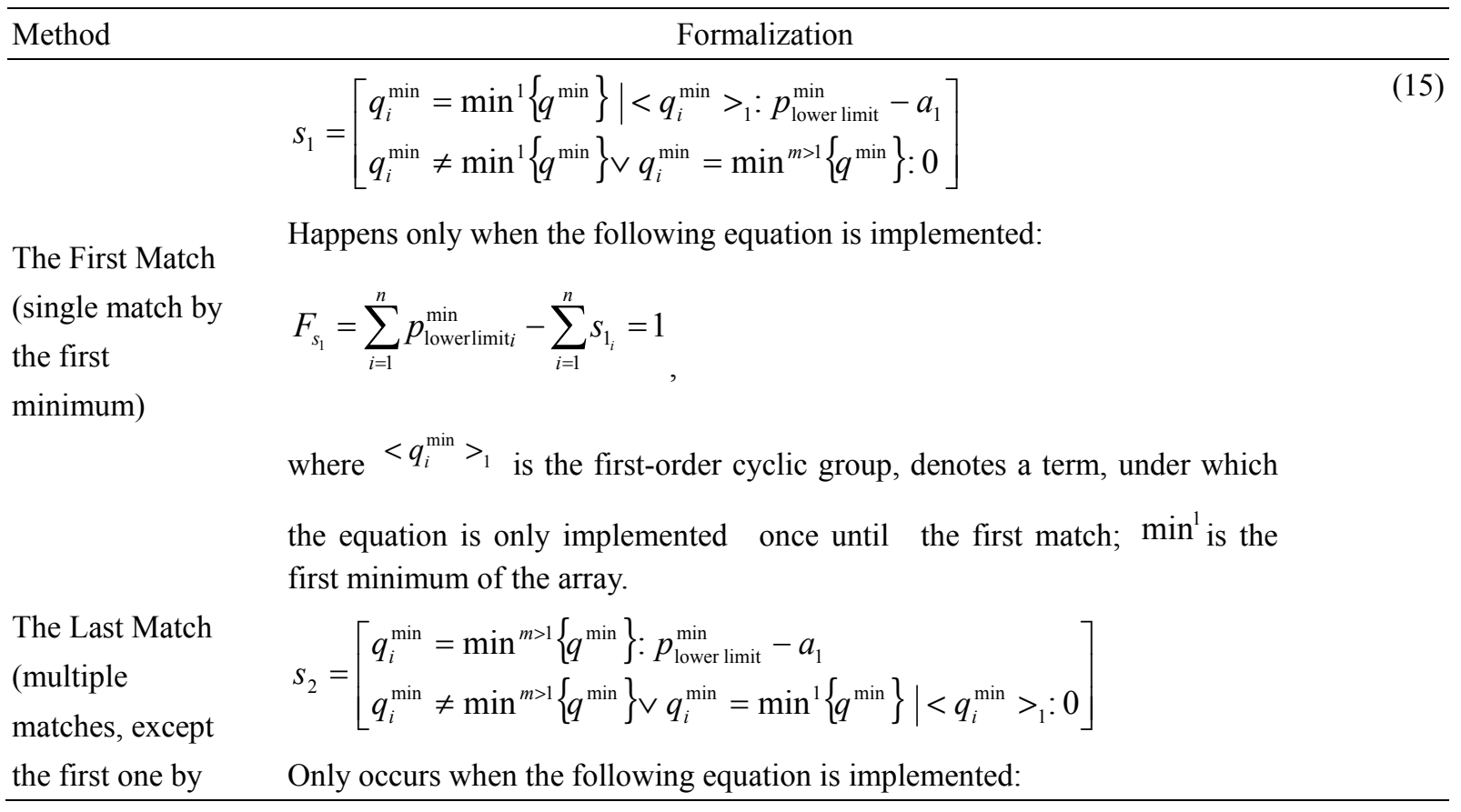




\begin{tabular}{|c|c|}
\hline Method & Formalization \\
\hline $\begin{array}{l}\text { the minimum } \\
\text { difference) }\end{array}$ & $F_{s_{2}}=\sum_{i=1}^{n} p_{\text {lowerlimiti } i}^{\min }-\sum_{i=1}^{n} s_{2_{i}}=1$ \\
\hline $\begin{array}{l}\text { The Inter } \\
\text { Matches } \\
\text { (matches by the } \\
\text { first, second etc. } \\
\text { minimum - till the } \\
\text { last included) }\end{array}$ & $\begin{array}{l}s_{3}=\left[\begin{array}{l}q_{i}^{\min }=\min ^{m<n}\left\{q^{\min }\right\} \vee \operatorname{Rank}_{i}\left(q_{i}^{\min }\right)<W_{q^{\min }}: p_{\text {lowerlimit }}^{\min }-a_{1} \\
q_{i}^{\min }=\min ^{m=n}\left\{q^{\min }\right\}: 0\end{array}\right. \\
\text { Only occurs only when the following equation is implemented: } \\
F_{s_{3}}=\sum_{i=1}^{n} p_{\text {lowerlimiti }}^{\min }-\sum_{i=1}^{n} s_{3_{i}}=1\end{array}$ \\
\hline
\end{tabular}

Each method seems to generate its proper solution. Moreover, as it was stated above, the choice is only made regarding one of the methods. Thus, the actual probability for the minimum uncertainty within the interval can be obtained as follows:

$$
\begin{aligned}
& p\left(H_{\text {real }}^{\min }\right)=\left[\begin{array}{l}
F_{s_{1}}=1 \mid<\{F\}>_{1}: p_{\text {lower limit } i}^{\min }-s_{1 i} \\
F_{s_{2}}=1 \mid<\{F\}>_{1}: p_{\text {lower limit } i}^{\min }-s_{2 i} \\
F_{s_{3}}=1 \mid<\{F\}>_{1}: p_{\text {lower limit } i}^{\text {min }}-s_{3 i}
\end{array}\right] \\
& \text { wherein } \sum_{i=1}^{n} p_{i}\left(H_{\text {real }}^{\min }\right)=1 \text {. }
\end{aligned}
$$

An outcome from the approach presented by the author is a set of two probability matrices, i.e. for the minimum (16) and for the maximum (9). Complexity and multiplicity of a logical search for the interval uncertainty depend on specifics in obtaining extrema for the function of the information entropy. To some extent, the challenge lies in hidden valuation approaches of the interval uncertainty. On the one hand, it has been taken for granted, i.e. environmental conditions under which an economic subject exists. However, on the other hand, it is sometimes not enough to be aware of fuzziness in the entropy distribution. Assumptions of certain disclosure of intervals as such are additional information actually not available to the subject. With absent properly elaborated approaches to evaluate the interval uncertainty (as opposed to approaches of mechanically selected values), such scientific objectives have been rent from the real life and oversimplified. We have mentioned above of a danger from simplification when obtaining the uncertainty and this reinforces relevance of this research.

On this occasion, it would be appropriate to refer to considerations made by Shary (2012), who in a definition for the interval uncertainty says of its important role for science. He comes to a fair conclusion that "the interval analysis and its specific methods have ... the highest significance in issues, where the uncertainty and ambiguity arise from the outset and are an integral part of task setting process".

\section{Discussion}

The interval analysis of the uncertainty in presented estimation methods puts a theoretical and methodological issue of applied usage of results. Contradictions, on the one hand, concern an objectively available natural uncertainty limit for the equiprobable outcome. On the other hand, the range of the interval uncertainty, as the wider the range of the minimum and maximum entropy is, the more the case approaches a status of one with a totally unknown outcome. This really causes displaced characteristics of the uncertainty, both with initial fuzziness of the system parameters, and their ultimate specificity. The obtained results for the interval estimation might relatively not highly efficient being applied in practice. The main cause for this is the mentioned uncertainty, but not related to a description of a choice situation as such, but to a confirmation of the "quality" of uncertainty in this choice. The mentioned methodological challenge was referred to as a challenge of uncertainty convergence. 


\subsection{Methodological Issue of Uncertainty Convergence}

Referring with more details to the issue, we need to explain its content. For the interval analysis, the uncertainty lies between the minimum and maximum limits. It can be conventionally called a baseline case. Nevertheless, boundaries of the uncertainty besides their calculated values have quite natural restrictions. The minimum uncertainty cannot obviously be below zero, while the maximum cannot be over the uncertainties for the equiprobable outcome. If a restraining barrier for the minimum uncertainty is neglected for a while, its maximum limit causes the convergence challenge. Indeed, the greater the probability range is, the greater the uncertainty range is. A further expanding probability only aggravates the case, where the lowest boundary of the interval has its shift. A logical consequence from this is a fact that the interval uncertainty cannot describe to a proper degree all the complexity and ambiguity of an expected choice.

At a turn of limit values for the minimum and maximum interval estimates, the uncertainty overlooks conceptual vagueness related to the uncertainty itself. Indeed, the uncertainty then does not allow making a reliable picture of a case. Its values fall in natural constraints, where there are descriptions for all possible options for a case to be resolved, even hardly probable. This emphasizes imperfection and limitations in theoretical and methodological solutions to the interval uncertainty. A logical conclusion suggests itself saying that its assessments, on the one hand, are only fair for certain ranges of probabilities for the ensemble of alternatives, thus confirming a conclusion of redundancy and pessimism in an array of ensured responses (according to Shary (2007). On the other hand, a new type of the uncertainty appear (like a technical parameter). Semantic formalization of such a conclusion can be expressed in the "uncertainty under uncertainty". It is there, where their convergence challenge focus on.

In either event, known approaches to solve this issue can be reduced to intervals convergence. It is worth saying that about at the same time many researchers addressed these issues. Thus, Ben-Nairn and Prade (2008) made an efficient attempt to solve a task of trust towards a particular economic agent with the generalized interval, "which necessarily describes the past behaviour [alongside existing behaviour patterns in the present time scholium]". But at the same time, a difficulty arises, i.e. whether such an interval is sufficiently compressed. There is another solution to the issue in the paper by Levin (2004), where he argues his stand of over fuzzy sets. However, the convergence issue cannot be solved by a simple combination of intervals or separation from them such a component, which locates at their intersection point.

\subsection{Tenversion Uncertainty}

Based on the abovementioned, we can say of a substitutive role of the additional uncertainty with respect to the interval one. To ease the reading, to the author's mind, its new type is appropriate to by referred to using a concept of the tenversion uncertainty (Note 5). It assumes describing how difficult it is to obtain the specific uncertainty among the interval range. At the same time, converging upper and lower bounds of the alternative probability reduces the tenversion uncertainty. As far as you get closer to the specific instance, it reduces to zero. For a clear description it would be appropriate to refer to the uncertainty function for the interval (see Figure 2 and 3).

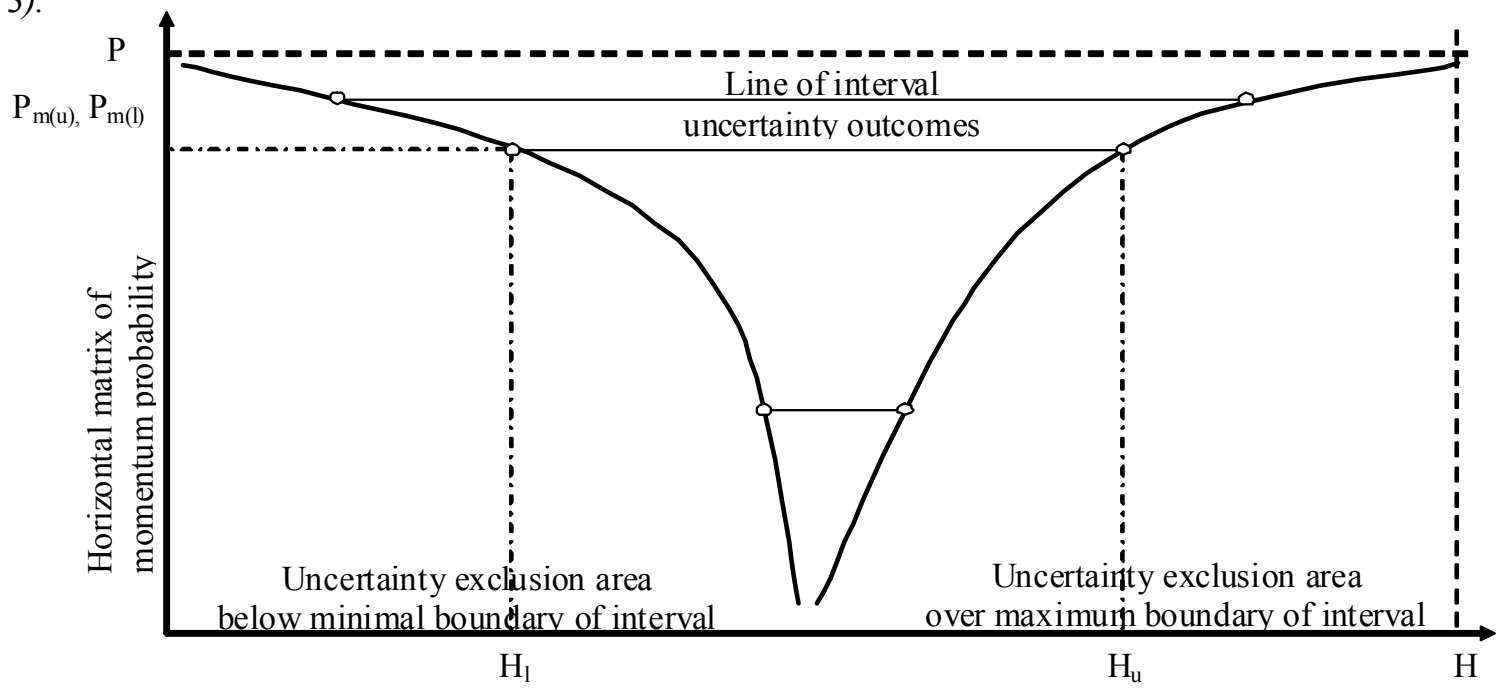

Figure 2. Function of the interval uncertainty for inhomogeneous alternatives

(where $\mathrm{H}$ is entropy, $\mathrm{P}$ is possibility, $\mathrm{Pm}$ is momentum possibility, l-low, u-upper). 


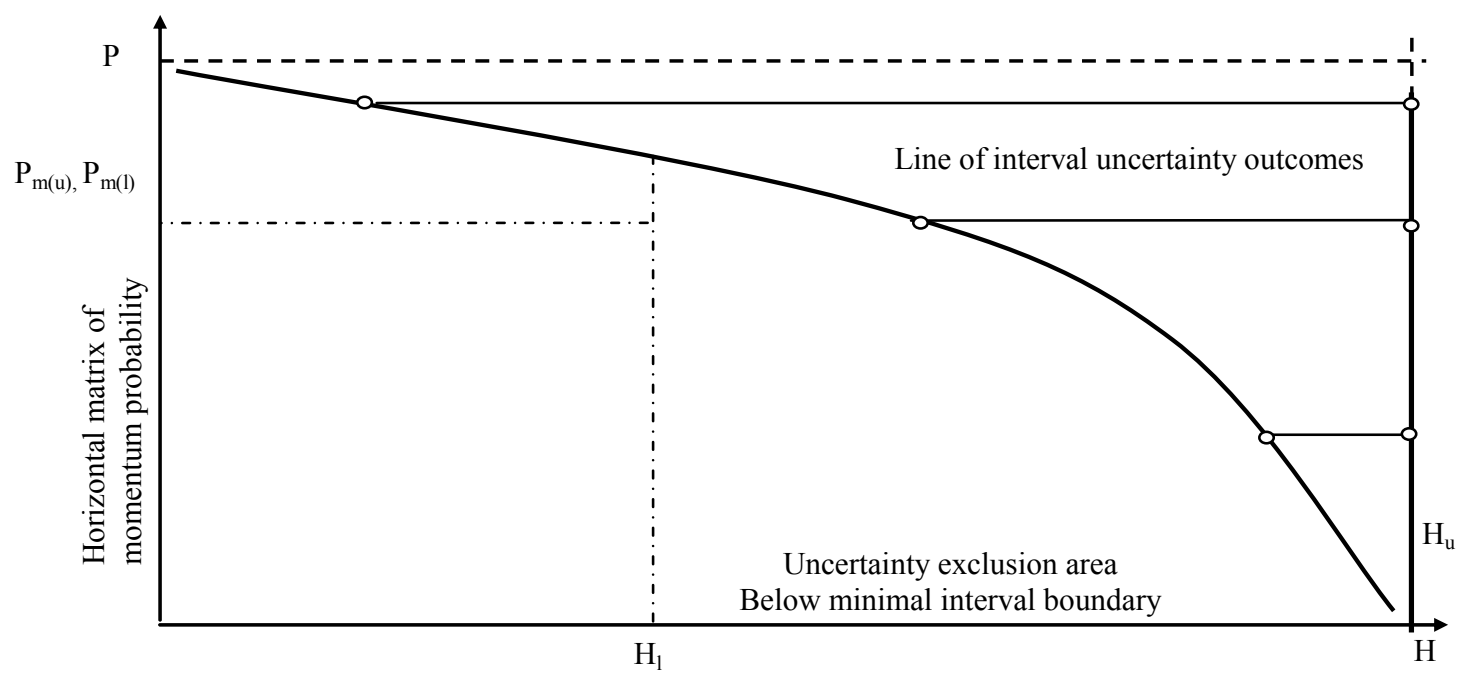

Figure 3. Function of interval uncertainty for homogeneous alternatives

(where $\mathrm{H}$ is entropy, $\mathrm{P}$ is possibility, $\mathrm{Pm}$ is momentum possibility, l-low, u-upper).

Being in a conjunction with the interval, the tenversion uncertainty has its quite reasonable limit equal to the maximum of the instance uncertainty, described in the interval analysis, except that a structure of alternatives gets its identically similar probability disregarding not specified range restrictions. Scientific consistency for this assumption is based on the so-called extreme case when the frequency range of source alternatives causes the uncertainty contained in natural constraints.

There have been no similar solutions to the issue of evaluating the tenversion uncertainty in known academic papers. Therefore, the author proposes an approach to calculate it. This suggests that such a task is put for the first time. The calculation method involves a removal of a dummy component from its structure. Its role is to collect "exact probabilities", i.e .those probabilities, which cannot be below the set case. All the hypotheses recently developed, must be similar at the same time. Hence, the probability remaining from the single instance (minus the "exact" one) is distributed between them. A calculation can be represented as follows:

$$
\begin{gathered}
H_{t}(I)=-\sum_{j=1}^{n} c_{j} \times \log _{b} c_{j} \\
c_{j}=\frac{a_{2}-a_{1}}{n}=\frac{1-\sum_{i=1}^{n} a_{1_{i}}}{n} \text { where } \sum_{j=1}^{n} c_{j}<1,
\end{gathered}
$$

where $H_{t}(I)$ is the tenversion uncertainty and $c_{j}$ is the equiprobable alternative under the tenversion uncertainty.

It may seem that a term when alternatives for the tenversion uncertainty do not constitute the single instance is erroneous. However, it seems be not the case. The dummy component complements the single instance, whereas the uncertainty depending on the lowest bound of the probability has been included in the interval uncertainty, even in a form of some wide range. As a result, there has remained a probability pool blurring clear boundaries of choice complexity and hence the uncertainty at time of such choice.

\section{Conclusion}

Summarizing the findings one can say for sure that the interval uncertainty is not only a characteristic form of the unknown, inexact and ambiguous in economic, but also in any other sphere of the social life. The environment is fuzzy and stochastic (probabilistic, - in research by Kim, Ovseyevich and Reshetnyak (1993), in a contrast to non-stochastic aspect - in papers by Elishako, Cai and Starnes (1994)), where solving certain administrative tasks has been reduced to optimization and anti-optimization techniques. It facilitates identifying aspects of the uncertainty previously unknown to scientists. The presented in the research new type of the uncertainty, called tenversion, in this context, is an illustrative example. The presented approach to logical evaluation of the interval uncertainty shows its balanced consistency in methods, their continuity in learning the 
experience gained in uncertainty studies. Many fundamental conclusions such as on probability averaging, accentuate available essential contradictions between set and conventionally established stereotypes. Further research would help making the uncertainty theory smoother in recently found specifics and essential manifestations.

\section{Acknowledgement}

The Russian Scientific Foundation (RSF), project No. 14-18-00574 "The Information and Analytical System Anti-Crisis: Region Diagnosis, Threat Assessment and Scenario Forecasting to Preserve and Strengthen Economic Security and Raise Welfare in Russia" supported this research.

\section{References}

Afanasyev, V. G. (1975). Social Information and Social Management. [Sotsial'naia informatsiia i upravlenie obshchestvom]. Moscow, Russia: Politizdat.

Ang, A. H. S., \& Tang, W. H. (1975). Probabilistic Concepts in Engineering Planning and Design. New York: John Wiley.

Avdiysky, V. I., \& Kuzmin, E. A. (2013). Criterion conditions to manifest stability in economics: indicative parameter of uncertainty. [Kriterial'nye usloviia proiavleniia ustoichivosti $\mathrm{v}$ ekonomike: indikativnyi parametr neopredelennosti]. Issues of Risk Analysis, 6(10), 10-22. Retrieved June 14, 2014, from http://elibrary.ru/item.asp?id= 21338344

Belyaev, L. S., \& Makarov, A. A. (1974). Uncertainty Factor in Making Optimal Decisions in Large Energy Systems, Vol. 2. [Faktor neopredelennosti pri priniatii optimal'nykh reshenii v bol'shikh sistemakh energetiki]. Novosibirsk, Russia: Siberian Energy Institute.

Ben-Nairn, J., \& Prade, H. (2008). Evaluating Trustworthiness from Past Performances: Interval-Based Approaches. In S. Greco, \& T. Lukasiewicz (Eds.), Scalable Uncertainty Management: Lecture Notes in Computer Science (Vol. 5291, pp. 33-46). Springer. http://dx.doi.org/10.1007/978-3-540-87993-0_5

Borisov, A. N., Alekseev, A. V., \& Merkureva, G. V., et al. (1989). Processing Fuzzy Information in Decision Making Systems. [Obrabotka nechetkoi informatsii v sistemakh priniatiia reshenii]. Moscow, Russia: Radio and Communication.

Chanas, S., \& Kamburowski, J. (1981). The use of fuzzy variables in PERT. Fuzzy Set Systems, 5, 1-19. http://dx.doi.org/10.1016/0165-0114(81)90029-4

Dubois, D., Prade, H., \& Smets, P. (1996). Representing partial ignorance. IEEE Transactions on Systems, Man and Cybernetics, 26, 361-377. http://dx.doi.org/10.1109/3468.487961

Elishako, I., Cai, G. Q., \& Starnes, J. H., Jr, (1994). Non-linear buckling of a column with initial imperfection via stochastic and non-stochastic convex model. International Journal of Non-Linear Mechanics, 29(1), 71-82. http://dx.doi.org/10.1016/0020-7462(94)90053-1

Elishakoff, I., \& Ohsaki, M., (2010). Optimization and Anti-optimization of Structures under Uncertainty. London, United Kingdom: World Scientific.

Fagin, R., \& Halpern, J. Y. (1991). A New Approach to Updating Beliefs, Uncertainty. In Bonissone, et al. (Eds.), Artificial Intelligence 6 (pp. 347-374). Elsevier Science Publishers.

Ferson, S., \& Ginzburg, L. (1996). Different methods are needed to propagate ignorance and variability. Reliability Engineering and Systems Safety, 54, 133-144. http://dx.doi.org/10.1016/S0951-8320(96)00071-3

Fortin, J., Zieliński, P., Dubois, D., \& Fargier, H., (2010). Criticality analysis of activity networks under interval uncertainty. Journal of Scheduling, 6 (13), 609-627. http://dx.doi.org/10.1007/s10951-010-0163-3

Guo, P., \& Tanaka, H., (2007). Interval regression analysis and its application. Ipm30: Interval and Imprecise Data Analysis. Proceedings of the 56th Session, Bulletin of the International Statistical Institute, Lisboa, Portugal.

Guo, P., \& Tanaka, H., (2010). On Probabilities Interval. In Huynh, V. N., Nakamori, Y., Lawry, J., \& Inuiguchi, M. (Eds.), Integrated Uncertainty Management and Applications. Springer. http://dx.doi.org/10.1007/978-3-642-11960-6_15

Halpem, J., \& Fagin, R. (1992). Two views of belief: Belief as generalized probability and belief as evidence. Artificial Intelligence, 54, 275-317. Retrieved June 14, 2014, from http://researcher.watson.ibm.com/researcher/files/us-fagin/ai92.pdf 
Hapke, M., Jaszkiewicz, A., \& Słowiński, R. (1994). Fuzzy project scheduling system for software development. Fuzzy Sets and Systems, 67, 101-107. http://dx.doi.org/10.1016/0165-0114(94)90211-9

Kelley, J. E. (1961). Critical path planning and scheduling-mathematical basis. Operations Research, 9, 296-320. http://dx.doi.org/10.1287/opre.9.3.296

Kim, Y. V., Ovseyevich, A. I., \& Reshetnyak, Y. N. (1993). Comparison of stochastic and guaranteed approaches to the estimation of the state of dynamic systems. Journal of Computer and System Sciences International, 31(6), 56-64. Retrieved June 14, 2014, from http://elibrary.ru/item.asp?id=12733744

Kreinovich, V., Wu, B., \& Xiang, G. (2012). Computing Statistics under Interval and Fuzzy Uncertainty: Applications to Computer Science and Engineering. Berlin, Germany: Springer. http://dx.doi.org/10.1007/978-3-642-24905-1

Kuzmin, E. A. (2012). Uncertainty and Certainty in Management of Organizational and Economic Systems. [Neopredelennost' i opredelennost' $\mathrm{v}$ upravlenii organizatsionno-ekonomicheskimi sistemami]. Ekaterinburg, Institute of Economics, Ural Branch of the RAS.

Kyburg, H. E., Jr. (1989). Higher Order Probabilities. In Kanai, L. N., Levitt, T. S., \& Lemmer, J. F. (Eds.), Uncertainty in Artificial Intelligence 3 (pp. 15-22). Elsevier Science Publishers, Amsterdam.

Leshkevich, T. (1994). Uncertainty in World and World of Uncertainty: Philosophical Reflections on Order and Chaos. [Neopredelennost' v mire i mir neopredelennosti: filosofskie razmyshleniia o poriadke i khaose]. Rostov-on-Don, Russia: Rostov University Press.

Levin, V. I. (2011). Simulating optimization tasks under interval uncertainty conditions. [Modelirovanie zadach optimizatsii v usloviiakh interval'noi neopredelennosti]. News of the V.G. Belinsky Penza State Pedagogical University, 26, 589-595. Retrieved June 14, 2014, from http://cyberleninka.ru/article/n/modelirovanie-zadach-optimizatsii-v-usloviyah-intervalnoy-neopredelennost i

Levin, V. I. (2004). Interval logic and over fuzzy sets. Theory and application. [Interval'naia logika i sverkhnechetkie mnozhestva. Teoriia i primenenie]. Bulletin of the Tambov State Technical University, 4-1(10), 924-929. Retrieved June 14, 2014, from http://www.vestnik.tstu.ru/rus/t_10/pdf/10_4_004.pdf

Li, H., \& Yue, D. (2010). Synchronization of Markovian jumping stochastic complex networks with distributed time delays and probabilistic interval discrete time-varying delays. Journal of Physics A: Mathematical and Theoretical, 43(10), 105101. http://dx.doi.org/10.1088/1751-8113/43/10/105101

Li, H., Wong, W. K., \& Tang, Y. (2012). Global Synchronization Stability for Stochastic Complex Dynamical Networks with Probabilistic Interval Time-Varying Delays. Journal of Optimization Theory and Applications, 2(152), 496-516. http://dx.doi.org/10.1007/s10957-011-9917-0

Lindley, D. V. (1987). The probability approach to the treatment of uncertainty in artificial intelligence and expert systems. Statistical Science, 2, 17-24. http://dx.doi.org/10.1214/ss/1177013427

Loostma, F. A. (1997). Fuzzy logic for planning and decision-making. Dordrecht: Kluwer Academic.

Loui, R. (1986). Interval-based decisions for reasoning systems. In L. N. Kanal, \& J. E. Lemmer (Eds.), Uncertainty in Artiicial Intelligence (pp. 459-472). Elsevier Science Publishers, Amsterdam, Netherlands. http://dx.doi.org/10.1016/B978-0-444-70058-2.50039-5

McCahon, C. S., \& Lee, E. S. (1988). Project network analysis with fuzzy activity times. Computers and Mathematics with Applications, 15, 829-838. http://dx.doi.org/10.1016/0898-1221(88)90120-4

Neapolitan, R. E. (1996). Is higher-order uncertainly needed? IEEE Transactions on Systems, Man and Cybernetics, 26, 294-302. http://dx.doi.org/10.1109/3468.487955

Nguyen, H. T., Kosheleva O., Kreinovich, V., \& Person, S. (2008). Trade-off between Sample Size and Accuracy: Case of Dynamic Measurements under interval uncertainty. In Huynh, V. N., Nakamori, Y., \& Ono, H. et al. (Eds.), Interval / Probabilistic Uncertainty and Non-classical Logics. Springer. http://dx.doi.org/10.1007/978-3-540-77664-2_5

Panyukov, A. V., \& Latipova, A. T. (2008). Evaluation of Equilibrium in von Neumann's Model with Interval Uncertainty of Source Data. [Otsenka ravnovesiia v modeli Neimana pri interval'noi neopredelennosti iskhodnykh dannykh]. Equilibrium Models in Economics and Energetics. Proceedings of the All-Russian Conference and Section of Mathematical Economics at the 14th Baikal International School-Workshop. Irkutsk, Russia: Energy Systems Institute of Siberian Branch of the Russian Academy of Sciences. 
Parsons, S., (2001). Qualitative Methods for Reasoning under Uncertainty. United States: MIT Press.

Parygin, B. D. (1978). Technological Revolution and Personality. [Nauchno-tekhnicheskaia revoliutsiia i lichnost']. Moscow, Russia: Politizdat.

Prade, H. (1979). Using fuzzy sets theory in a scheduling problem: a case study. Fuzzy Sets and Systems, 2, 153-165. http://dx.doi.org/10.1016/0165-0114(79)90022-8

Rommelfanger, H. (1994). Network analysis and information flow in fuzzy environment. Fuzzy Sets and Systems, 67, 119-128. http://dx.doi.org/10.1016/0165-0114(94)90212-7

Shang, Y. (2011). Robustness of scale-free networks under attack with tunable grey information. EPL (Europhysics Letters), 95(2), 28005. http://dx.doi.org/10.1209/0295-5075/95/28005

Shary, S. P. (2007). Interval analysis or Monte Carlo methods? [Interval'nyi analiz ili metody Monte-Karlo?]. Computational Technologies, 1(12), 103-115.

Shary, S. P. (2012). Finite Measured Interval Analysis. [Konechnomernyi interval'nyi analiz]. Novosibirsk, Russia: Institute of Computational technologies of SB RAS; XYZ Press.

Shen, B., Wang, Z., Ding, D., \& Shu, H., (2013). $H_{\infty}$ state estimation for complex networks with uncertain inner coupling and incomplete measurements. IEEE Trans Neural Netw Learn Syst. 24(12), 2027-2037. http://dx.doi.org/10.1109/TNNLS.2013.2271357

Spall, J. C. (2002). Uncertainty Bounds in Parameter Estimation with Limited Data. In Dror, M., L'Ecuyer, P., \& Szidarovszky, F. (Eds.), Modeling Uncertainty: an Examination of Stochastic Theory, Methods, and Applications (pp. 685-710). Springer. http://dx.doi.org/10.1007/0-306-48102-2_27

Sternin, M., \& Shepelyov, G., (2012). Evaluating interval alternatives: uncertainties and preferences. [Otsenka interval'nykh al'ternativ: neopredelennosti i predpochteniia]. Information Models and Analyses, 1, 357-369. Retrieved June 14, 2014, from http://www.foibg.com/ijima/vol01/ijima01-4.pdf

Xiang, G. (2007). Fast Algorithms for Computing Statistics under Interval Uncertainty, with Applications to Computer Science and to Electrical and Computer Engineering. United States: The University of Texas at El Paso. Computer Science; ProQuest.

Yager, R. R., \& Kreinovich, V. (1999). Decision making under interval probabilities. International Journal of Approximate Reasoning Qualitative, 22, 195-215. http://dx.doi.org/10.1016/S0888-613X(99) 00028-6

\section{Notes}

Note 1. It is quite reasonable that a view of averaging incorrectness assumes an opposition between the uncertainty nature and framing of that alternative among many similar, which would make a picture of its dominating over others, as it is such alternative, which comes out of a total ensemble of hypotheses. Averaging only applies to "free" alternatives; therefore, remaining may differ from the others. Then the uncertainty will be relatively less, as a case of choice is clearer. However, it is difficult to say definitely that the averaging method cannot be used. On the contrary, an example of the equiprobable outcome shows that the method is reasonable to maximize the uncertainty. A mixed option implies separating alternatives, their differentiation into fixed and mobile (free) hypothesis in a probabilistic manifestation. Hence, a significant assumption occurs, in which a set of alternatives is only partially homogeneous; therefore, fixed alternatives appear as dominating. Similar ideas validating the averaging one can trace in the paper by Levin (2011), who says of existing optimization approach based on average values of the system parameters, enclosed within the interval value.

Note 2. Saying the "first match", the author assumes identifying such an alternative, for which for the first time there will be met additional requirements regardless whether there are similar or exactly one-to-one hypothesis of analogous dimensions in intervals of probabilities. The first match is intended to reveal the alternative that should degrade an overall layout of the uncertainty in favour of probability reducing by, on the one hand, comparing uncertainties of the lowest and upper thresholds, and, on the other hand, by calculating a difference between them, that will be a criterion of such a hypothesis found. We should clarify that the "first match" has been accepted as one of possible methods. It is not excluded and it is even allowed to choose any other serial match of a minimum difference of boundary uncertainties between alternatives, if any. It is important for such a match of two or more to be exclusive.

Note 3. Saying the method of "the last match" the author refers to logical and arithmetic operations with those alternatives that: firstly, are identically one-to-one and similar to each other; secondly, they cover all the 
matching alternatives except the first one. It is worth saying that with synectic similar of alternatives, a choice of the first one is based on identification simplicity, while it is not excluded and it is allowed to choose another serial alternative from a set of matching subject to logical conditions for exception and other related operations to harmonize probabilities and make the single instance.

Note 4. For the third option, a calculation of the $q^{\text {min }}$ indicator according to the equation (12) has retained its original form, with an exception that values of the applied probability are changed. Herewith a decrease (probability change) each time occurs by the same value. Logical values of the error according to (12) assume identifying those alternatives, a change to the probability of which is impossible.

Note 5. An origin for a concept of the "tenversion uncertainty" goes back to Latin terms "tension" (tension), "tenus" (up to) and "vaste" (broad, wide). In the author's opinion, the tenversion uncertainty complements the interval evaluation with the new informative content. It seems that the probability range causes a case when the interval uncertainty loses complexity of choice identification across the uncertainty's full length. It seems that the tenversion uncertainty solves this issue, essentially reflecting the interval filling.

It is worth paying attention that the tenversion uncertainty is not included in a cyclic sequence of a change to types of the environmental uncertainty, decision-making, consequences of these decisions and the vartational uncertainty. It is important to understand that the tenversion uncertainty accompanies the interval analysis, confirming or non-confirming its acceptability for each specific case.

\section{Copyrights}

Copyright for this article is retained by the author(s), with first publication rights granted to the journal.

This is an open-access article distributed under the terms and conditions of the Creative Commons Attribution license (http://creativecommons.org/licenses/by/3.0/). 